Klein M, Alwazzan D, Chakraborty N.

A Direct Numerical Simulation analysis of pressure variation in turbulent premixed Bunsen burner flames-Part 1: Scalar gradient and strain rate statistics.

Computers and Fluids 2018

DOI: https://doi.org/10.1016/j.compfluid.2018.03.010

\section{Copyright:}

(C) 2018. This manuscript version is made available under the CC-BY-NC-ND 4.0 license

DOI link to article:

https://doi.org/10.1016/j.compfluid.2018.03.010

Date deposited:

$21 / 03 / 2018$

Embargo release date:

10 March 2019

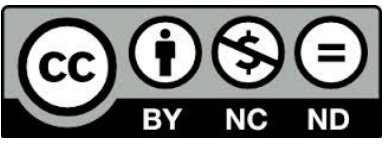

This work is licensed under a

Creative Commons Attribution-NonCommercial-NoDerivatives 4.0 International licence 
and Fluids

Elsevier Editorial System(tm) for Computers

Manuscript Draft

Manuscript Number: CAF-D-17-00845R1

Title: A Direct Numerical Simulation analysis of pressure variation in turbulent premixed Bunsen burner flames-Part 1: Scalar gradient and strain rate statistics

Article Type: SI:29TH PARCFD CONFERENCE(Marco Fossati)

Keywords: Premixed flame, Bunsen burner flame, Pressure, Direct Numerical Simulation

Corresponding Author: Professor Markus Klein, Ph.D.

Corresponding Author's Institution: Universität der Bundeswehr München

First Author: Markus Klein, Ph.D.

Order of Authors: Markus Klein, Ph.D.; Dana Alwazzan; Nilanjan Chakraborty, Ph.D.

Abstract: Three-dimensional simple chemistry Direct Numerical Simulations (DNS) of Bunsen burner flames have been carried out for different pressure values. A number of cases have been considered for the same set of values of mean and root-mean-square inlet velocities normalised by the laminar burning velocity and the integral length scale normalised by the nozzle diameter. The modifications of laminar burning velocity and flame thickness with pressure lead to an increase in both flow and turbulent Reynolds numbers with increasing pressure. This also gives rise to changes in Damköhler number and Karlovitz numbers for these flames and thus they occupy different locations on the regime diagram. For this reason, two additional cases at the lowest pressure have been simulated to match the turbulent Reynolds number of the highest-pressure case by changing the normalised root-mean-square velocity in one case, whereas the integral length scale is modified in the other case. It has been found that pressure and turbulent Reynolds number variations do not have significant influences on the mean behaviours of the magnitude of the reaction progress gradient (i.e. Surface Density Function) and fluiddynamic normal strain rate. However, the length scale separation between the nozzle diameter and flame thickness increases with increasing pressure, which makes the occurrence of the Darrieus-Landau (DL) instability highly likely for the flames at elevated pressures. The presence of the DL instability affects the flame curvature statistics, which in turn influence the mean behaviours of the dilatation rate and fluid-dynamic tangential strain rate. 
Universität der Bundeswehr

Universität der Bundeswehr München · 85577 Neubiberg · Germany

Computers \& Fluids Editorial Board

Dr. Marco Fossati
Fakultät für Luft- und Raumfahrttechnik Institut für Mathematik und Rechneranwendung

Univ.-Prof. Dr.-Ing. habil. Markus Klein

$\begin{array}{ll}\text { Telefon } & +49896004-2122 \\ \text { Telefax } & +49896004-2135 \\ \text { E-Mail } & \text { markus.klein@unibw.de } \\ & \text { 6. Februar 2018 }\end{array}$

\section{Paper Submission}

Dear Dr. Fossati,

please find attached the revised version of our paper entitled "A Direct Numerical Simulation analysis of pressure variation in turbulent premixed Bunsen burner flames-Part 1: Scalar gradient and strain rate statistics "

authored by M. Klein, D. Alwazzan and N. Chakraborty submitted to the special issue of the $29^{\text {th }}$ PARCFD conference.

We have addressed all the reviewer's comments in a rebuttal and the revised version of the manuscript, except for his suggestion to combine part 1 and part 2 of the paper in one single article. This was impossible because of the page limit and we followed your kind advice in this regard. Thanks for all the support during submission and review! We look forward to hearing from you in due course.

With best regards (on behalf of all authors)

Markus Klein 


\section{A Direct Numerical Simulation analysis of pressure variation in turbulent premixed Bunsen burner flames-Part 1: Scalar gradient and strain rate statistics}

\section{Reviewer \#1:}

This is an interesting and well written paper studying the effect of pressure variation on turbulent premixed Bunsen burner flames. I have a few minor corrections/comments and one major suggestion, which I list below. If all these are satisfactorily addressed than I can recommend the acceptance of the paper for publication.

We thank the reviewer for his positive recommendation and for his useful remarks. We addressed all remarks carefully in the revised version of our manuscript and provided an appropriate answer in this rebuttal. For the reviewers convenience all changes are shown in a red font.

\section{1) Page 2, Section 3. In the definition of the turbulent Reynolds number a definition for " $I$ " is not given, please define.}

The turbulent length scale $l$ is the longitudinal integral length scale $L_{11}$ of the inflow turbulence prescribed at the nozzle. This is now explicitly mentioned on p.2 in the manuscript.

2) Page 3, Section 3. It is stated that three different grids are used for the simulation of the various cases. A justification for the usage of these grids is needed and a grid convergence study or/and relevant references should also be added.

The domain is $2 D \times 2 D \times 2 D$ in all cases where the dimension of $D$ remains unchanged. Apart from the inflow boundary all other boundaries are outflows and it has been found that the domain is large enough to avoid undesirable interaction of the flame or turbulence with the boundaries.

Grid convergence studies are in the context of DNS rather unusual because almost always impossible. The grid spacing is chosen in such a way that the Kolmogorov scale and the flame thickness can be resolved. Burner geometry and domain size have been considered fixed after that, but as the thermal flame thickness decreases with increasing pressure (i.e. $\delta_{t h} \sim p^{-0.5}$ ) the grid spacing has to follow exactly the same trend. This gives exactly rise to the grid dimensions mentioned below in question 3 (e.g. $795 / 250 \approx \sqrt{10}$ ).

All the above information is now provided on p. 3 of the revised paper.

It is also worth noting that the required resolution depends on the question under consideration, i.e. the answer is relative. As an example if one is interested in the tails of the PDFs of sixth order moments a finer grid spacing is needed (even smaller than Kolomogorov scale) compared to first and second order moment statistics. For this reviewer's convenience, we show the resolution of the laminar flame profile with the grid spacing used in the manuscript and with half the grid spacing (i.e. doubled grid size). Furthermore, as this is a central quantity in the present work we show a conditional plot of SDF (surface density function) $|\nabla c|$. The figure shown below indicates that the results can for all practical purposes be considered identical. 

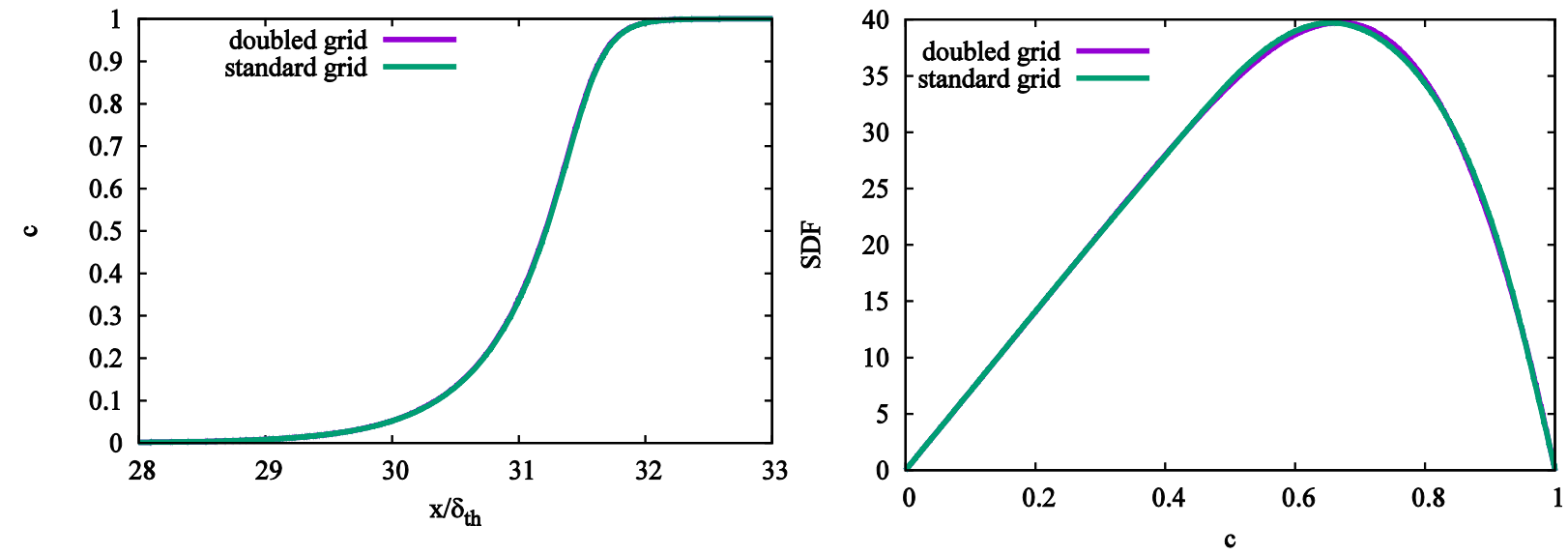

The following table gives the ratio of the estimated Kolmogorov scale (based on the turbulent Reynolds number) and the grid spacing. It is clearly seen that the Kolmogorov scale is in all cases resolved.

\begin{tabular}{|c|c|c|c|c|c|}
\hline Case & A & B & C & D & E \\
\hline $\boldsymbol{\eta} / \boldsymbol{\Delta x}$ & 3.59 & 4.44 & 4.86 & 1.54 & 4.86 \\
\hline
\end{tabular}

We believe that all these information will be a distraction in the paper and thus are only presented in the rebuttal but the underlying message is conveyed on p. 3 of the revised paper.

3) Page 3, Section 3. A short scaling study is reported but with different grids than those used for the analysis runs. Was there a specific reason why the authors used them instead of the $256^{\wedge} 3,560^{\wedge} 3$ and $795^{\wedge} 3$ grids?

As explained in question 2 the grid size for the (different pressure) Bunsen flames are dictated by physics. The grids for the scaling analysis (which was done independent of the analysis of Bunsen flames) have been determined based on two principles: (i) the SUPERMUC system has a minimum and a maximum number of nodes that can be used on a particular architecture according to a given set of rules. For the scaling analysis shown e.g. in Fig. 2a the minimum number of processors was 512. (ii) In order to get a nice scaling curve with several points it must be possible to equally distribute the grid onto all processors used. It appeared that $(3 \cdot 360)^{3}=1080^{3}$ is a good choice because it can be distributed on $8^{3}, 10^{3}, 15^{3}, 20^{3}, 24^{3}, 27^{3}$ processes. This information is provided on pp. 3-4 of the revised paper.

4) Page 3, Figure 2a. A percentage showing the deviation from the ideal scaling could be added next to each point.

We followed the reviewer's suggestion. The achieved parallel performance for the $10^{3}, 15^{3}, 20^{3}, 24^{3}, 27^{3}$ grids is $95 \%, 90 \%, 79 \%, 74 \%, 66 \%$ respectively. This information is provided on p. 4 of the revised paper. 


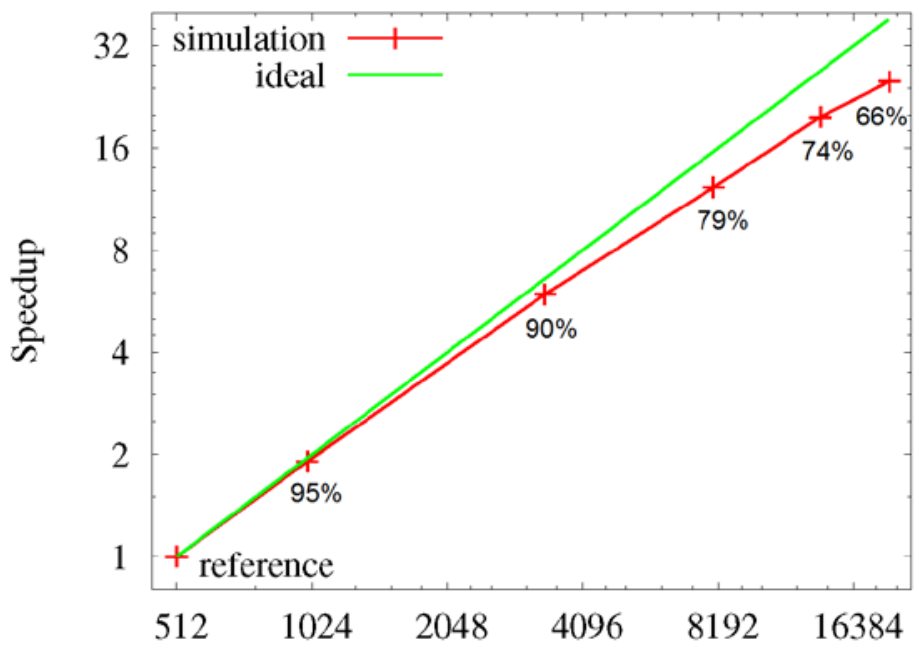

5) Figures 4, 5, 6, 7, 8 and 9 could have only one part combining (a) and (b) reducing the size of paper and giving a better comparison between the different cases.

This reviewer's idea is good in principle but will work only for a part of the figures (e.g. Figs. 5 and 7), whereas lines are so close to each other that it will be difficult to combine them in one plot for Figs. 4,6,8 and 9. As an example, Fig. 4 with cases A-E combined is shown below for the convenience of this reviewer:

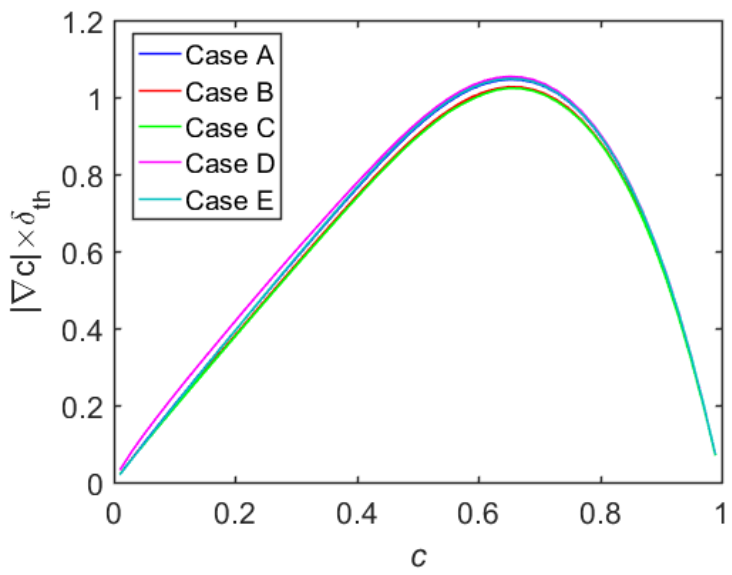

The series of Figures 4-9 is consistently split in cases A,B,C (where pressure changes but inlet turbulence has been kept constant) and cases C,D,E (where inlet parameters of 1 bar cases D,E, are adjusted to yield the same Reynolds number as 10 bar case C). We would prefer to keep this in a consistent manner. However, if this reviewer insists, we will be ready to change the figures.

6) Figure 10 can show one case only and state in the text that all cases exhibit similar qualitative behaviours.

We agree with the reviewer and followed his/her advice (see p. 9 of the revised paper). 


\section{7) Page 10, Section 4.5, line 3 the dot over " $w /<$ rho $>$ " should be placed over " $w "$.}

We have corrected this typographical error (see p. 9 of the revised paper).

\section{8) Reference 1, the title should be corrected to "Scalar dissipation rate and flame..."}

We thank the reviewer for pointing this out and corrected the typographical error (see p. 11 of the revised paper)..

9) This paper can be combined with part 2 and make a more complete contribution. Many parts of both papers are almost identical (introduction, mathematical background, numerical implementation, etc.) while results of this paper are already discussed in part 2. The readers would benefit if all the results and corresponding discussion are in one paper.

We mostly agree with this reviewer and before submission we actually had prepared one single paper. The editor of the special issue explained to us that there is a strict page limit of 12 pages. That is the reason why the paper was split into two parts according to the suggestion of the special issue editor (this was clearly stated at time of submission in the cover letter as well).

After receiving this review we asked the editor if it will be possible to extent the page limit such that we can combine both parts of the paper in one contribution and in fact we would have been prepared for that because the paper initially was written as a single article. However, it will be entirely impossible to combine two 12 page papers into a single 12 page paper even after considerable shortenings. The email was send to Prof. Sagaut and to SI editor Dr. Fossati. The reply was:

"I suggest you keep the two parts separated and try to make clear in the abstract / introduction of both manuscripts the split nature of your contribution that leads to some unavoidable repetitions."

We have followed this suggestion and in order to make the paper self-contained it is unavoidable to repeat information like mathematical background and numerical implementation and this is explained on pp. 2-4 of Part 2. Following the reviewers comment we changed the wording in sections "Mathematical Background" and "Numerical Implementation" on pages 2-3 of the revised version part 2. The continuation of the analysis on the SDF transport is also explicitly indicated on p. 10 of Part 1. 


\section{HIGHLIGHTS}

1. Parametric analysis of high pressure Bunsen burner flames using DNS

2. Mean values of scalar gradient and strain rate are unaffected by pressure change

3. High pressure flames are likely to exhibit the Darrieus-Landau (DL) instability

4. The DL instability affects dilatation rate and tangential strain rate statistics

5. Modelling implications for elevated pressure flames have been discussed 


\title{
A Direct Numerical Simulation analysis of pressure variation in turbulent premixed Bunsen burner flames-Part 1: Scalar gradient and strain rate statistics
}

\author{
M. Klein ${ }^{\mathrm{a} *}$, D. Alwazzan ${ }^{\mathrm{b}}$, N. Chakraborty \\ aUniversität der Bundeswehr München, Fakultät für Luft- und Raumfahrtechnik, 85577 Neubiberg \\ bSchool of Mechanical \& Systems Engineering, Newcastle University, Newcastle-Upon-Tyne, NE17RU
}

\begin{abstract}
Three-dimensional simple chemistry Direct Numerical Simulations (DNS) of Bunsen burner flames have been carried out for different pressure values. A number of cases have been considered for the same set of values of mean and root-mean-square inlet velocities normalised by the laminar burning velocity and the integral length scale normalised by the nozzle diameter. The modifications of laminar burning velocity and flame thickness with pressure lead to an increase in both flow and turbulent Reynolds numbers with increasing pressure. This also gives rise to changes in Damköhler number and Karlovitz numbers for these flames and thus they occupy different locations on the regime diagram. For this reason, two additional cases at the lowest pressure have been simulated to match the turbulent Reynolds number of the highest-pressure case by changing the normalised root-mean-square velocity in one case, whereas the integral length scale is modified in the other case. It has been found that pressure and turbulent Reynolds number variations do not have significant influences on the mean behaviours of the magnitude of the reaction progress gradient (i.e. Surface Density Function) and fluid-dynamic normal strain rate. However, the length scale separation between the nozzle diameter and flame thickness increases with increasing pressure, which makes the occurrence of the Darrieus-Landau (DL) instability highly likely for the flames at elevated pressures. The presence of the DL instability affects the flame curvature statistics, which in turn influence the mean behaviours of the dilatation rate and fluiddynamic tangential strain rate.
\end{abstract}

Keywords:

Premixed flame, Bunsen burner flame, Pressure, Direct Numerical Simulation

\section{INTRODUCTION}

The statistical behaviour of the magnitude of the gradient of reaction progress variable $|\nabla c|$ plays a key role in fundamental understanding and modelling of turbulent premixed combustion. For example, the probability density function of burning mixture [1] and pocket formation in premixed flames [2] can be analysed in terms of $|\nabla c|$ statistics. The magnitude of the gradient of reaction progress variable $|\nabla c|$ is often termed as the Surface Density Function (SDF) [2] due to its close relation to flame surface area and generalised Flame Surface Density (FSD) $\Sigma_{g e n}=\overline{|\nabla c|}$ [3], where the overline represents either Reynolds averaging or filtering operation, as applicable. Furthermore, the SDF is closely related to scalar dissipation rate $\left(\mathrm{SDR}=N_{c}=D|\nabla c|^{2}\right.$ where $D$ is the progress variable diffusivity) of reaction progress variable. The evolution of SDF has been studied by several authors [4-9] and the strain rate and curvature dependences of the SDF and its transport equation terms have been analysed in detail. The alignment of $\nabla c$ with local principal strain rates in turbulent premixed flames, and its implication on the FSD and SDR transport have been addressed by Chakraborty and Swaminathan and their co-workers [10-12]. Recently, Dopazo and coworkers [13-17] derived transport equations for the normal distance between two adjacent $c$ isosurfaces and linked this transport equation to the transport equation of the SDF. A recent study of Wang et al. [18] analysed the statistics of the SDF and the strain rates affecting its

\footnotetext{
* Corresponding Author

Email address: markus.klein@unibw.de
}

transport using Direct Numerical Simulation (DNS) data of a high-Karlovitz number jet flame. The analyses reported in Refs. [4-9,13-18] revealed the close relation of the SDF $|\nabla c|$ with fluid-dynamic strain rates and flame displacement speed $S_{d}$. It is worth noting that most of the aforementioned analyses (e.g. [4,5,7-11,13,15-18]) have been carried out in canonical configurations. Moreover, all the aforementioned analyses have been carried out for atmospheric pressure. In many engineering applications (e.g. Spark Ignition engines, industrial gas turbines) combustion takes place at elevated pressures and thus it is essential to assess if the statistical behaviours of the SDF, fluid-dynamic strain rates and displacement speed in turbulent premixed flames at elevated pressure conditions remain qualitatively similar to the corresponding statistics for atmospheric flames. This motivated the current analysis which focuses on the analysis of SDF, strain rate and displacement speed statistics of turbulent premixed flames in Bunsen-burner configuration for different pressure values. In hydrocarbon-air mixtures the laminar burning velocity and flame thickness decrease with increasing pressure and thus flame resolution for a fixed geometry becomes more challenging for higher pressures. Here, a single-step Arrhenius type chemical mechanism is used for the purpose of computational economy and also in order to isolate the fluid-dynamical effects of pressure variation. The main objectives of this paper are: 
(a) To analyse the statistics of normal and tangential strain rates in turbulent premixed flames for a Bunsen burner configuration for different pressure values.

(b) To provide detailed explanations for the observed pressure dependences of strain rates and SDF.

(c) To indicate the implications of the above statistics on the modelling of premixed turbulent flames at elevated pressures.

To meet the aforementioned objectives, three flames (i.e. cases A-C with increasing pressure from case A to case C) have been considered for three different pressure values but the inlet values of mean and root-mean square (rms) velocities normalised by laminar burning velocity and integral length scale of turbulence normalised by nozzle diameter are kept unaltered in analogy to the situation in a real Bunsen flame burner. As viscosity and hence flame thickness and burning velocity change with pressure, a change in pressure for a given flow condition leads to a modification of the turbulent Reynolds number and the position of the flame on the combustion regime diagram. In addition to these three flames, two additional cases (i.e. cases $\mathrm{D}$ and $\mathrm{E}$ ) at reference pressure have been considered which have the same turbulent Reynolds number as the one of the elevated pressure flame (i.e. case C), and this is achieved by modifying the normalised rms turbulent velocity fluctuation for one of the cases (i.e. case D), whereas in the other case (i.e. case E) the ratio of integral length scale to flame thickness has been modified. These three cases (i.e. cases C-E) with same turbulent Reynolds number help to isolate the effects of pressure variation.

The rest of the paper will be organised as follows. The mathematical background and numerical implementation pertaining to this analysis are presented in the next two sections. The results will be presented next and finally, the main findings will be summarised and conclusions will be drawn.

\section{MATHEMATICAL BACKGROUND}

The current analysis considers a generic single-step Arrhenius type irreversible chemical mechanism for the purpose of computational economy due to the demands of flame resolution at elevated pressures, and also owing to the fact that a Bunsen burner configuration is a relatively complex geometry for DNS. Moreover, DNS with detailed chemical mechanism remains extremely expensive [19] for a detailed parametric analysis as conducted in this paper. For hydrocarbon-air combustion the unstrained laminar burning velocity $S_{L}$ decreases with pressure $P$ as: $S_{L} \sim P^{-0.5}$ [20], whereas dynamic viscosity $\mu$ does not change with pressure but ideal gas density $\rho$ increases with pressure as $\rho \propto P$. This implies that the thermal flame thickness $\delta_{t h}=\left(T_{a d}-T_{0}\right) / \max |\nabla T|_{L}$ (where $T, T_{0}$ and $T_{a d}$ are the instantaneous dimensional, unburned gas and adiabatic flame temperatures respectively) scales as: $\delta_{t h} \sim \mu /\left(\rho S c S_{L}\right) \sim P^{-0.5}$. In the context of a simple chemical mechanism, the pre-exponential factor and kinematic viscosity have been altered to account for the desired pressure dependence. Thus, the present analysis focuses only on the fluid-dynamical aspects of the pressure dependence.

The transport equation of the magnitude of reaction progress variable gradient $|\nabla c|$ in the reference frame attached with the flame are given as [13-17]:

$\frac{1}{|\nabla c|} \frac{d|\nabla c|}{d t}=-\left(a_{N}+N_{j} \frac{\partial S_{d}}{\partial x_{j}}\right)$ or, $\frac{\partial|\nabla c|}{\partial t}+\frac{\partial\left(v_{j}|\nabla c|\right)}{\partial x_{j}}=\left(a_{T}+2 S_{d} \kappa_{m}\right)|\nabla c|$

Here, $d(\ldots) / d t=\partial(\ldots) / \partial t+v_{j} \partial(\ldots) / \partial x_{j}$ with $v_{j}=$ $u_{j}+S_{d} N_{j}$ being the $\mathrm{j}^{\text {th }}$ component of the flame propagation velocity. The $\mathrm{j}^{\text {th }}$ component of fluid velocity, flame normal vectors and flame displacement speed are given by $u_{j}, N_{j}=-\left(\partial c / \partial x_{j}\right) /|\nabla c|$ and $S_{d}$ respectively. The quantities $a_{N}=N_{i} N_{j} \partial u_{i} / \partial x_{j}$ and $a_{T}=\left(\delta_{i j}-\right.$ $\left.N_{i} N_{j}\right) \partial u_{i} / \partial x_{j}$ are fluid-dynamic normal and tangential strain rates respectively and $\kappa_{m}=0.5\left(\partial N_{i} / \partial x_{i}\right)$ is the arithmetic mean of two principal curvatures. According to the current convention the flame normal points towards the reactants and the flame surface convex to the reactants has a positive curvature. The flame displacement speed $S_{d}$ is defined as [21,22]:

$S_{d}=\frac{\dot{w}+\nabla \cdot\left(\rho D_{c} \nabla c\right)}{\rho|\nabla c|}=S_{r}+S_{n}+S_{t}$

where

$S_{r}=\frac{\dot{w}}{\rho|\nabla c|} \quad S_{n}=\frac{\vec{N} \cdot \nabla\left(\rho D_{c} \vec{N} . \nabla c\right)}{\rho|\nabla c|} \quad S_{t}=-2 D_{c} \kappa_{m}$

Here $\dot{w}$ and $D_{c}$ are the reaction rate of reaction progress variable and mass diffusivity of the reaction progress variable, and $S_{r}, S_{n}$ and $S_{t}$ are the reaction, normal diffusion and tangential diffusion components of displacement speed, respectively [21,22].

It can be seen from eqs. 1 and 2 that the statistical behaviours of $|\nabla c|, a_{T}, a_{N}$ and $S_{d}$ are closely related [1317] and thus these statistics will be discussed in detail in Section 4 of this paper for flames with different pressure values.

\section{NUMERICAL IMPLEMENTATION}

A well-known DNS code SENGA [23] has been used for the simulations carried out in this analysis. In SENGA, the conservation equations of mass, momentum, energy and reaction progress variables are solved in non-dimensional form. The spatial discretisation has been carried out using a $10^{\text {th }}$ order central difference scheme but the order of accuracy gradually drops to a $2^{\text {nd }}$ order one-sided scheme at the non-periodic boundaries. The time-advancement has been carried out using a low-storage $3^{\text {rd }}$ order Runge-Kutta scheme. The values of flow Reynolds number $R e=$ $U_{B} D / v_{u}$ based on the bulk inlet velocity $U_{B}$, nozzle diameter $D$, and the kinematic viscosity in the unburned gas $v_{u}$, turbulent Reynolds number $R e_{t}=u^{\prime} L_{11} / v_{u}$ normalised turbulent root-mean-square (rms) velocity fluctuation $u^{\prime} / S_{L}$, normalised inlet velocity $U_{B} / S_{L}$, longitudinal integral length scale to thermal flame thickness ratio $L_{11} / \delta_{t h}$, longitudinal integral length scale to nozzle diameter ratio $L_{11} / D$, Damköhler number $D a=$ $L_{11} S_{L} / \delta_{t h} u^{\prime}$, and Karlovitz number $K a=\left(u^{\prime}\right)$ $\left.S_{L}\right)^{3 / 2}\left(L_{11} / \delta_{t h}\right)^{-1 / 2}$ are given in Table 1 . The longitudinal integral length scale $L_{11}$ refers to the integral length scale of inflow turbulence prescribed at the nozzle. The heat release parameter $\tau=\left(T_{a d}-T_{0}\right) / T_{0}$ and the Zel'dovich number $\beta=T_{a c}\left(T_{a d}-T_{0}\right) / T_{a d}^{2}$ are taken to be 4.5 and 6.0 respectively where $T_{a c}$ is the activation temperature. Standard values of Prandtl number ( $\mathrm{Pr}=$ $0.7)$ and ratio of specific heats $\left(\gamma_{g}=1.4\right)$ have been used. The reference pressure $P_{0}$ is taken to be $1.0 \mathrm{bar}$ for the current analysis. All non-dimensional numbers mentioned before have to be understood as inlet values here and in 
the remainder of the text. It can be seen from Table 1 that cases A-C have same inlet values of $u^{\prime} / S_{L}, U_{B} / S_{L}$ and $L_{11} / D$ but $R e_{t}, D a$ and $K a$ values are different, and thus they fall at different locations on the combustion regime diagram as shown in Fig. 1 . The cases A-C and $E$ fall on the boundary of the wrinkled and the corrugated flamelets regimes according to the regime diagram by Peters [24] (see Fig. 1). Moreover, it can be noted from Table 1 that the turbulent Reynolds number $R e_{t}$ increases from case $\mathrm{A}$ to case $\mathrm{C}$. Furthermore, it can be seen from Table 1 that cases $C$, $\mathrm{D}$ and $\mathrm{E}$ have same values of $R e_{t}$ but cases $\mathrm{D}$ and $\mathrm{E}$ have one tenth of the pressure of that of case $C$. The value of $u^{\prime} / S_{L}$ is higher in case $\mathrm{D}$ than in case $\mathrm{C}$ and $\mathrm{E}$, whereas $u^{\prime} / S_{L}$ and $L_{11} / \delta_{t h}$ values are exactly the same for cases $\mathrm{C}$ and $\mathrm{E}$ and thus they fall on the same point on the regime diagram, as can be seen from Fig. 1. The simulation domain is taken to be $2 D \times 2 D \times 2 D$ which corresponds for cases A, D and E [case B] (case C) to a cube of $50 \delta_{t h} \times 50 \delta_{t h} \times 50 \delta_{t h}\left[112 \delta_{t h} \times 112 \delta_{t h} \times\right.$ $\left.112 \delta_{t h}\right] \quad\left(159 \delta_{t h} \times 159 \delta_{t h} \times 159 \delta_{t h}\right)$ which is discretised using a uniform Cartesian grid of $250 \times 250 \times 250 \quad[560 \times 560 \times 560](795 \times 795 \times 795)$ points, which ensures resolution of both the Kolmogorov length scale and the flame thickness.

\begin{tabular}{|c|c|c|c|c|c|c|c|c|c|c|}
\hline Case & $\boldsymbol{P} / \boldsymbol{P}_{\mathbf{0}}$ & $\boldsymbol{R} \boldsymbol{e}_{\boldsymbol{D}}$ & $\boldsymbol{R} \boldsymbol{e}_{\boldsymbol{t}}$ & $\boldsymbol{U}_{\boldsymbol{B}} / \boldsymbol{S}_{\boldsymbol{L}}$ & $\boldsymbol{u}_{\text {inlet }}^{\prime} / \boldsymbol{S}_{\boldsymbol{L}}$ & $\boldsymbol{L}_{\mathbf{1 1}} / \boldsymbol{D}$ & $\boldsymbol{L}_{\mathbf{1 1}} / \boldsymbol{\delta}_{\boldsymbol{t h}}$ & $\boldsymbol{K a}$ & $\boldsymbol{D a}$ & $\boldsymbol{\tau}$ \\
\hline $\mathrm{A}$ & 1.0 & 399 & 13.30 & 6.0 & 1.0 & $1 / 5$ & 5.20 & 0.45 & 5.00 & 4.5 \\
\hline $\mathrm{B}$ & 5.0 & 892 & 29.26 & 6.0 & 1.0 & $1 / 5$ & 11.40 & 0.30 & 11.40 & 4.5 \\
\hline $\mathrm{C}$ & 10.0 & 1262 & 41.22 & 6.0 & 1.0 & $1 / 5$ & 16.13 & 0.25 & 16.13 & 4.5 \\
\hline $\mathrm{D}$ & 1.0 & 399 & 41.22 & 6.0 & 3.1 & $1 / 5$ & 5.20 & 2.40 & 1.670 & 4.5 \\
\hline $\mathrm{E}$ & 1.0 & 399 & 41.22 & 6.0 & 1.0 & $3 / 5$ & 16.13 & 0.25 & 16.13 & 4.5 \\
\hline
\end{tabular}

Table 1: Attributes of inlet turbulence for the cases considered here

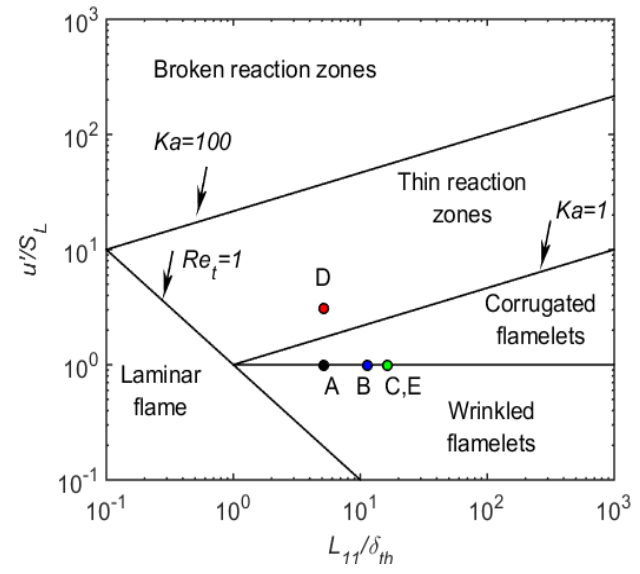

Figure 1: The cases considered here on the combustion regime diagram by Peters [24].

It is worth noting that the domain is $2 D \times 2 D \times 2 D$ in all cases where the dimension of $D$ remains unchanged. Apart from the inflow boundary all other boundaries are outflows and it has been found that the domain is large enough to avoid undesirable interaction of the flame or turbulence with the boundaries. The grid spacing is chosen in such a way that the Kolmogorov scale and the flame thickness can be resolved. As burner geometry and domain size have been considered fixed, the grid spacing has to decrease with increasing pressure as the thermal flame thickness decreases with an increase in pressure (i.e. $\delta_{t h} \sim p^{-0.5}$ ). It is almost impossible to carry out gridindependence analysis in a true sense but for the meshes chosen for this analysis the Kolmogorov length scale remains at least twice of the grid spacing. Moreover, halving the grid spacing did not have any noticeable impact on $|\nabla c|$ distribution (e.g. maximum difference is less than $1 \%$ ) for laminar flames.

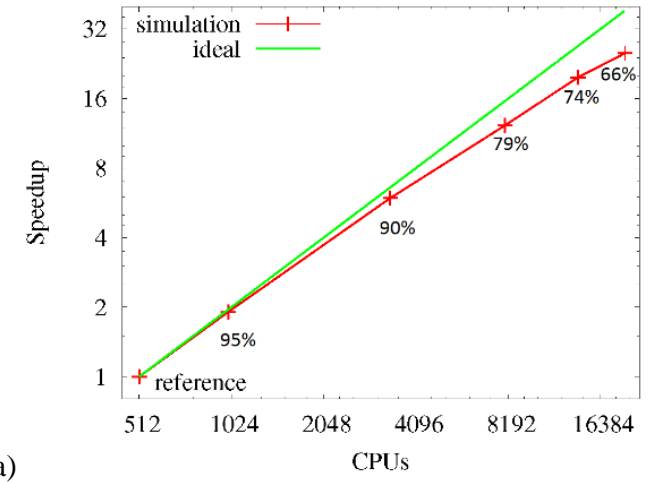

(a)

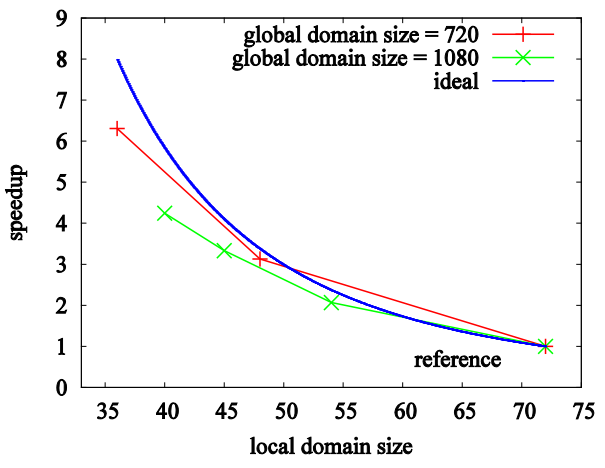

Figure 2: (a) Measured versus ideal speedup on a computational domain consisting of $1080^{3}$ grid points versus the number of CPUs; (b) Measured versus ideal speedup on domains consisting of $720^{3}$ and $1080^{3}$ grid points versus local domain size.

The code has been parallelised using MPI and the results of a scaling analysis are shown in Fig. 2a. The grid size has been chosen to get a nice scaling with several points, to allow for equal distribution of the grid on all processors and to ensure conformity with architecture usage rules. It appeared that $(3 \cdot 360)^{3}=1080^{3}$ is a good choice because it can be distributed on 
$8^{3}, 10^{3}, 15^{3}, 20^{3}, 24^{3}, 27^{3}$ processes. The achieved parallel performance for the $10^{3}, 15^{3}, 20^{3}, 24^{3}, 27^{3}$ grids is $95 \%, 90 \%, 79 \%, 74 \%, 66 \%$ respectively.

The speedups relative to the local domain size for global uniform grids of $720^{3}$ and $1080^{3}$ are shown in Fig. 2b, demonstrating that the parallel efficiency depends on the number of CPUs in total as well as the local (i.e. per CPU) grid size. It is worth noting that for a local grid size of $45^{3}$ the number of internal grid points is roughly the same as the number of grid points that are communicated for a $10^{\text {th }}$ order central difference scheme, i.e. $55^{2} \times 5 \times 6$. It can be seen that the parallel efficiencies starts to deteriorate gently if the local grid size is reduced from $45^{3}$.

Turbulent inflow data has been generated using a modified version of the method suggested by Klein et al. [25] based on digital filtering. The idea of the digital filter based generation of inflow data suggested in [25] is to filter random data in order to obtain realistic pseudo-turbulent velocity correlations. Combined with a coordinate transformation it is possible to prescribe local length scales as well as first and second order one-point statistics. As pointed out in Jarrin et al. [26] this method can become computationally expensive on fine meshes combined with large length scales, i.e. large filter sizes, if the original implementation is used. In order to overcome the efficiency problems related to the generation of synthetic turbulent inflow data on large distributed grids, several modifications have been implemented:

1. The Gaussian filter in temporal direction has been replaced by an autoregressive AR1 process requiring only two time levels, in order to avoid excessive filter length in this direction. This step is particularly suitable for compressible flow solvers because of the small time step values (see Ref. [27]).

2. The tensor product of the two-dimensional filter kernel remaining after optimisation step 1 can be replaced by two one-dimensional filters. Thus, the complexity of the filtering operation for a single grid point can be reduced from $O\left(N^{2}\right)$ to $O(N)$, where $N$ is related to the length of the filter in one direction [28].

3. In a straightforward implementation of the method proposed by Klein et al. [25], the communication of random data would be required before the filtering step takes place. However, message passing can be entirely avoided [28] by using identical random seeds for generating inflow data in buffer regions that overlap with neighbouring local domains.

4. Instead of filtering the inflow data for each local domain located in the inflow plane with its allocated CPU, the filtering can alternatively be done by all available processors. However, this requires communication and redistribution of the data in the inflow plane. The benefit of this step will depend on the relative time required for filtering and communication.

By following the sequence of steps 1-4 a very efficient generation of inflow data can be obtained. The time required for generating the inflow data has been found to be of the order of $1 \%$ of the time required for advancing one time step when all the above measures are combined.

Apart from inlet boundary, all the other boundaries of the computational domain are taken to be partially nonreflecting which are specified according to Navier Stokes
Characteristic Boundary Conditions (NSCBC) technique [29]. The reacting flow field is initialised by an unstrained premixed laminar flame solution which is specified as a function of radius from the nozzle center resulting in hemispherical scalar field located at the inflow. As in jet like flows the mean velocity profile after the nozzle exit has been approximated by a hyperbolic-tangent like distribution. The statistics have been extracted after 2 through-pass times (i.e. $2 L / U_{\text {mean }}$ where $L$ is the length of the simulation domain) and at least 10 different realisations have been utilised to extract the statistics.

\begin{tabular}{|c|c|c|c|}
\hline Case & $\boldsymbol{P} / \boldsymbol{P}_{\mathbf{0}}$ & $\langle\boldsymbol{S}\rangle$ & $\boldsymbol{S D}(\boldsymbol{S})$ \\
\hline A & 1.0 & 4.15 & 0.313 \\
\hline B & 5.0 & 4.46 & 0.34 \\
\hline C & 10.0 & 4.39 & 0.135 \\
\hline D & 1.0 & 4.13 & 0.43 \\
\hline E & 1.0 & 3.85 & 0.20 \\
\hline
\end{tabular}

Table 2: Normalised flame surface area $S=\int_{V}|\nabla c| d V /$ $D^{2}$ for the cases considered here. Here $\langle S\rangle$ and $S D(S)$ are the mean and standard deviation of $S$ based on different realisations.

\section{RESULTS \& DISCUSSION}

\section{1 flame-turbulence interaction}

The isosurfaces of reaction progress variable $c$, distributions of $c$ and normalised axial velocity component $u_{1} / S_{L}$ in the central mid-plane for cases A-E are shown in Fig. 3. Although cases A-C have the same set of values of $U_{B} / S_{L}, u^{\prime} / S_{L}$ and $L_{11} / D$ at the inlet, the flame morphology is considerably different for these three cases. From a visual inspection of Fig. 3 , it is clear that the nature of flame wrinkling is different between cases A-C. It is especially evident from Fig. 3 that the probability of finding sharply curved cusps increases from case A to case C. Moreover, the flame morphology is significantly different in cases C-E in spite of having the same turbulent Reynolds number $R e_{t}$. For example, the flame in case $\mathrm{E}$ is less wrinkled in comparison to that in case $\mathrm{C}$ in spite of having the same position on the regime diagram (see Fig. 1) based on the inlet turbulence. The higher value of $u^{\prime} / S_{L}$ in case $\mathrm{D}$ than in case $\mathrm{E}$ leads to a greater extent of flame wrinkling in case D in comparison to case E. The mean and standard deviation values of normalised flame surface area $S$ based on different realisations of cases A-E are listed in Table 2 where $S$ is evaluated by the volume integral given by: $S=\int_{V}|\nabla c| d V / D^{2}$. Table 2 shows that the mean normalised flame surface areas for cases A-C remain close to each other. Furthermore, the difference in mean values are much smaller than the standard deviations between different realisations so the pressure dependence on the normalised flame surface area is not prominent for cases A-C. A greater extent of flame wrinkling in case C (case $\mathrm{D}$ ) than case $\mathrm{E}$ is reflected in the form of higher mean flame surface area for case C (case D) reported in Table 2. The normalised axial velocity component $u_{1} / S_{L}$ exhibits high magnitudes at the flame tip where focussing of heat gives rise to strong thermal expansion, which, in turn, leads to flow acceleration near the flame tip. As $|\nabla c|$ plays a key role in determining flame area and flame thickness, the statistical behaviour of SDF and the terms which affect its evolution will be discussed next in this paper. 

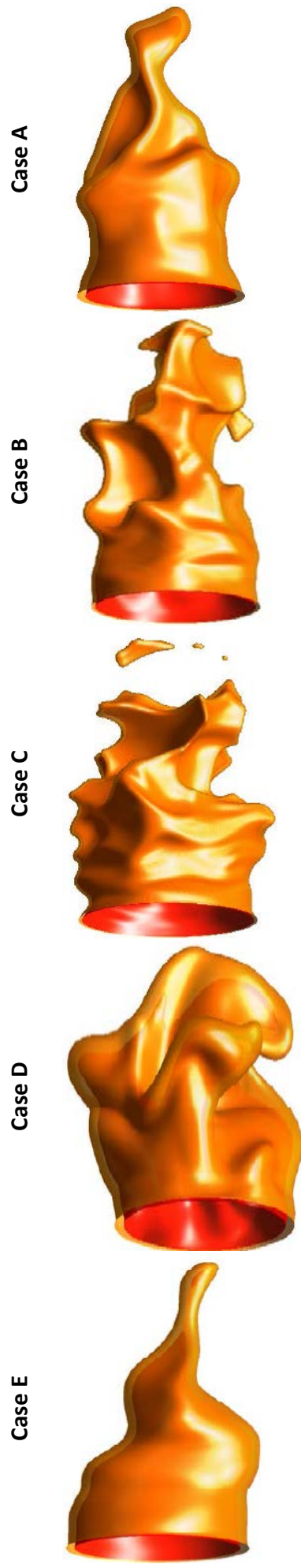

(a)
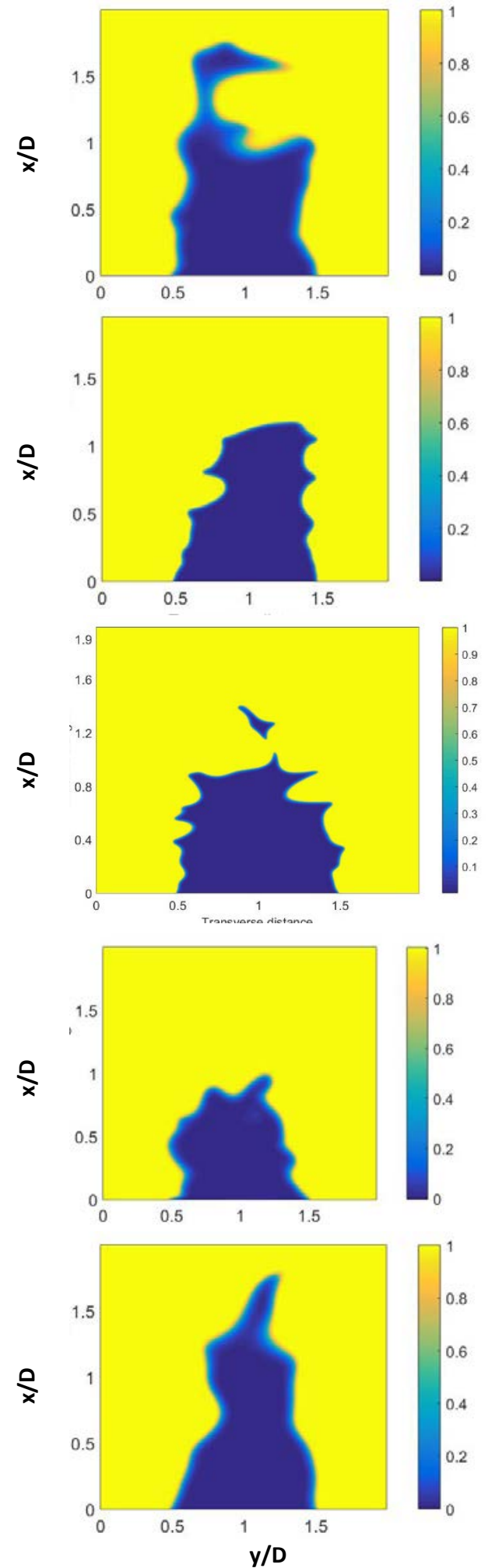

(b)
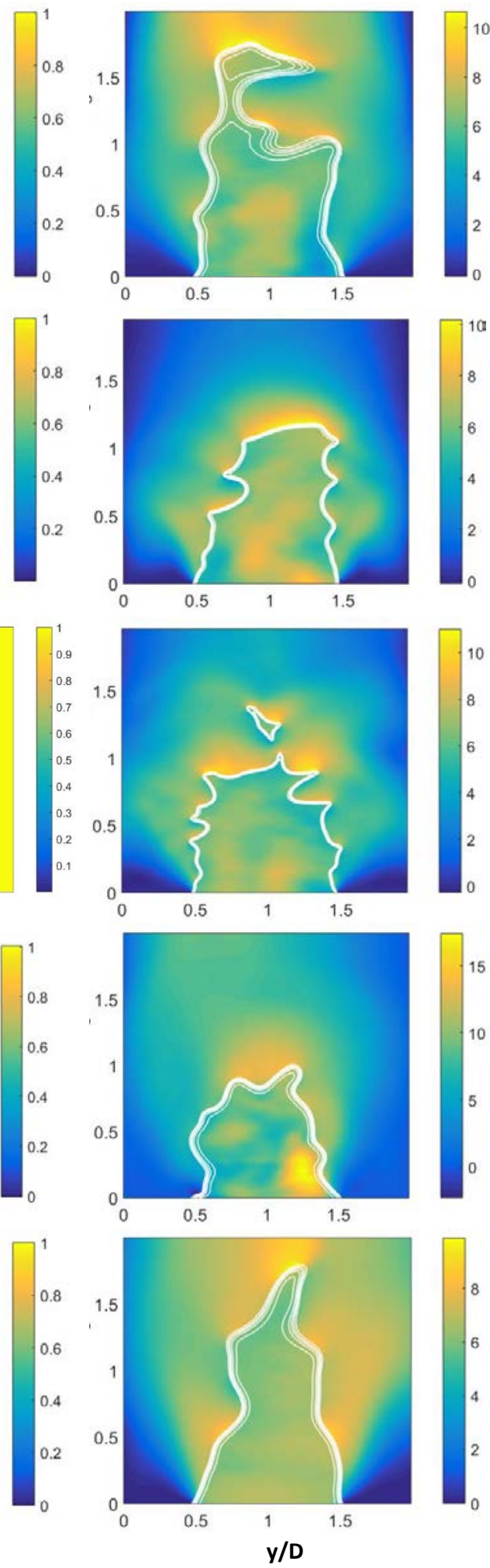

(c)

Figure 3: (a) Instantaneous view of isosurfaces of reaction progress variable $c\left(1^{\text {st }}\right.$ column); colour changes from red to orange from the unburned gas to the burned gas; (b) distributions of $c$ ( ${ }^{\text {nd }}$ column) and (c) normalised axial velocity component $u_{1} / S_{L}$ ( $3^{\text {rd }}$ column) in the central mid-plane for cases A-E. 
flame are shown in Fig. 4a for cases A-C. The corresponding variations for cases $\mathrm{C}-\mathrm{E}$ are shown in Fig. 4b. It is worth noting that the maximum value of $|\nabla c|$ scales as $|\nabla c| \sim 1 / \delta$, where $\delta$ is the local flame thickness. For low Mach number unity Lewis number globally adiabatic flames $c$ becomes identical to the nondimensional temperature $\theta=\left(T-T_{0}\right) /\left(T_{a d}-T_{0}\right)$ and thus $\delta_{t h}$ can be expressed as: $\delta_{t h}=1 / \max |\nabla c|_{L}$. Thus, the maximum value of $|\nabla c| \times \delta_{t h}$ provides a measure of the ratio of laminar flame thickness to turbulent flamelet thickness. A peak conditional mean value of $|\nabla c| \times \delta_{t h}$ with magnitude greater (smaller) than unity indicates a case of flame thinning (flame thickening) under turbulence in a mean sense. It is evident from Figs. 4a and $4 \mathrm{~b}$ that the peak mean value of $|\nabla c| \times \delta_{t h}$ conditional upon $c$ remains marginally greater than unity, which suggests that the flamelet thickness decreases marginally in comparison to laminar flame thickness in these cases. This flame thinning under turbulence is in accordance with experimental findings of Soika et al. [30] and DNS results by Hawkes et al. [31]. However, other experimental [3234] and DNS [6] findings reported flame thickening under turbulence.

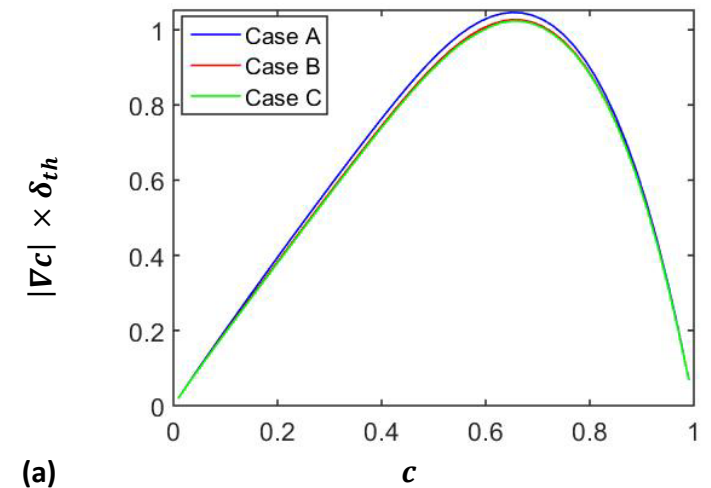

(a)

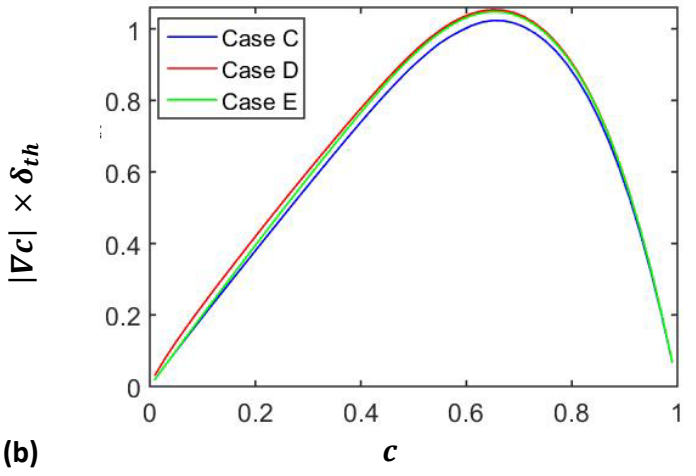

Figure. 4: Profiles of the mean values of normalised SDF $|\nabla c| \times \delta_{t h}$ conditional upon $c$ for cases (a) A-C and (b) CE.

It can be seen from Figs. 4a and 4b that there is little difference between the profiles of mean values of $|\nabla c| \times$ $\delta_{t h}$ conditional upon $c$ for cases A-E. It has been found that the standard deviations of $|\nabla c| \times \delta_{t h}$ conditional upon $c$ are much bigger than the difference in the profiles of the mean values of normalised SDF (not shown here for brevity). Thus, the small differences in mean profiles cannot be attributed to the variations of pressure and turbulent Reynolds number for the parameter range considered here. It is evident from eqs. 1 and 2 that both fluid-dynamic strain rates (i.e. $a_{N}$ and $a_{T}$ ), and flame displacement speed statistics play pivotal roles in the evolution of the SDF. For this reason, the behaviours of fluid-dynamic strain rates (i.e. $a_{N}$ and $a_{T}$ ), and flame displacement speed will be discussed next in this paper.

4.3 Statistical behaviour of fluid-dynamic strain rates The variations of mean values of normalised fluiddynamic normal strain rate $a_{N} \times \delta_{t h} / S_{L}$ conditional upon $c$ using the samples for the whole flame are shown in Fig. 5 a for cases A-C. The corresponding profiles for cases C$\mathrm{E}$ are shown in Fig. 5b. It is evident from Fig. 5 that the mean normal strain rate $a_{N}$ remains positive for all these cases, which indicates that fluid-dynamic normal strain rate tends to increase the distance between isoscalar surfaces and thus acts to reduce $|\nabla c|$.
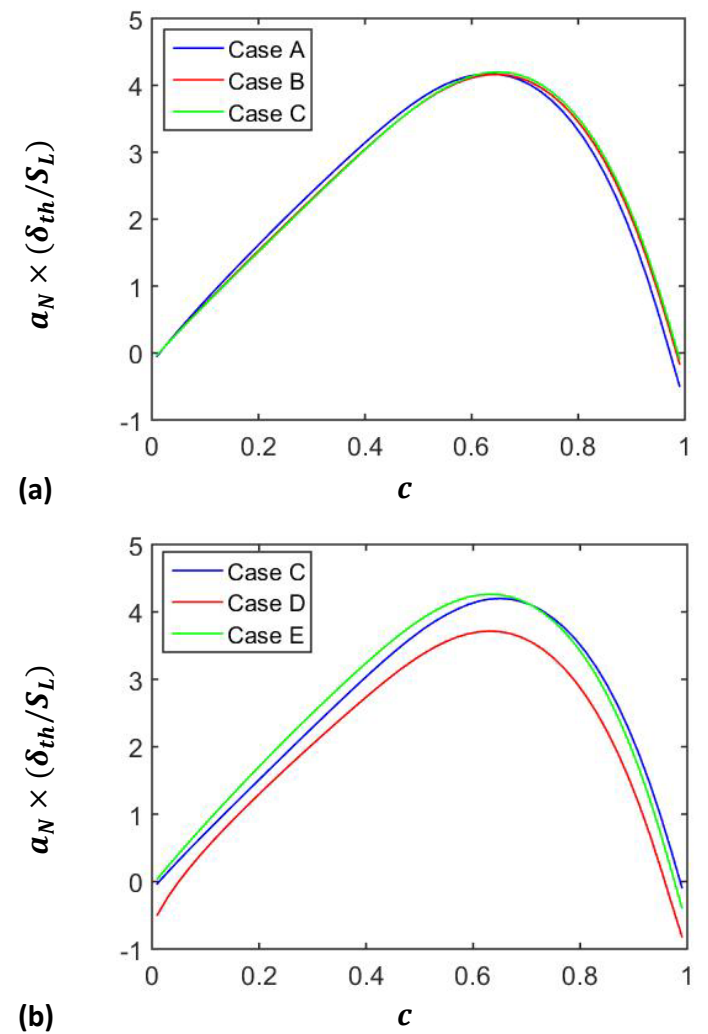

Figure. 5: Profiles of the mean values of normalised fluiddynamic normal strain rate $a_{N} \times \delta_{t h} / S_{L}$ conditional upon $c$ for cases (a) A-C and (b) C-E.

The fluid-dynamic normal strain rate is given by: $a_{N}=$ $\left(e_{\alpha} \cos ^{2} \alpha+e_{\beta} \cos ^{2} \beta+e_{\gamma} \cos ^{2} \gamma\right)$ where $e_{\alpha}, e_{\beta}$ and $e_{\gamma}$ are the most extensive, intermediate and the compressive principal strain rates respectively and $\alpha, \beta$ and $\gamma$ are the angles between $\nabla c$ with the eigenvectors associated with $e_{\alpha}, e_{\beta}$ and $e_{\gamma}$ respectively. It has been discussed elsewhere [5,10-12] that $\nabla c$ aligns predominantly with the most extensive principal strain rate for high Damköhler number (i.e. $D a>1$ ) combustion where the strain rate induced by chemical heat release dominates over turbulent straining. A preferential collinear alignment of $\nabla c$ with $e_{\alpha}$ leads to positive mean values of $a_{N}$ in all cases because combustion takes place under high $D a$ values in these flames (see Table 1). The profiles of mean values of $a_{N} \times$ $\delta_{t h} / S_{L}$ remain almost identical to each other in cases A-C 
and the standard deviations conditional upon $c$ (not shown here for conciseness) are larger than the differences in the mean values for these flames. The profiles of normalised mean fluid-dynamic normal strain rate $a_{N} \times \delta_{t h} / S_{L}$ for cases $\mathrm{C}$ and $\mathrm{E}$ are also close to each other and the standard deviations of $a_{N} \times \delta_{t h} / S_{L}$ conditional upon $c$ remain greater than the difference between the mean values so no physical effects can be attributed to this difference. Thus, a change in pressure does not have much influence on the mean behaviour of normalised fluid-dynamic normal strain rate $a_{N} \times \delta_{t h} / S_{L}$.

The mean value of $a_{N} \times \delta_{t h} / S_{L}$ for case $\mathrm{D}$ is smaller than that obtained for other cases considered here and the standard deviations between the realisations are not sufficient to neglect this difference. Case D has the smallest value of $D a$ among all the cases considered here and thus the extent of $\nabla c$ alignment with the most extensive principal strain rate is smaller than that in other cases because the strain rate due to thermal expansion is relatively weaker than turbulent straining in case D in comparison to other cases [10].
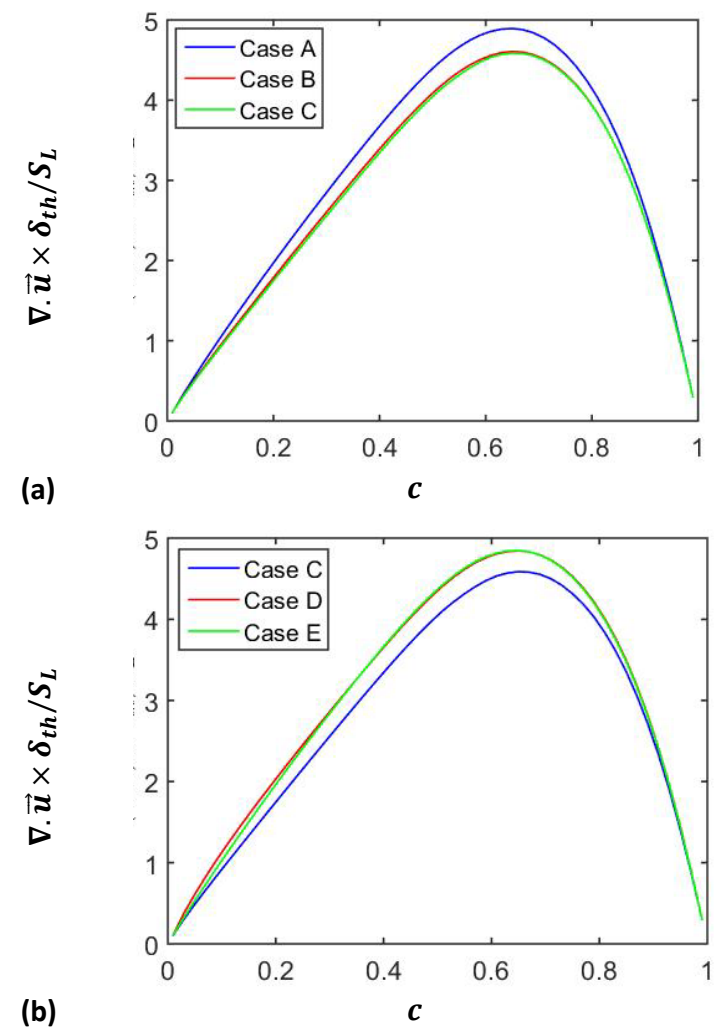

Figure 6: Profiles of the mean values of normalised dilatation rate $\nabla . \vec{u} \times \delta_{t h} / S_{L}$ conditional upon $c$ for cases (a) A-C and (b) C-E.

The variations of mean values of normalised dilatation rate $\nabla . \vec{u} \times \delta_{t h} / S_{L}$ conditional upon $c$ using the samples for the whole flame are shown in Figs. 6a and 6b for cases A-C and C-E respectively. It can be seen from Fig. 6a that the mean value of $\nabla \cdot \vec{u} \times \delta_{t h} / S_{L}$ in case $\mathrm{A}$ assumes greater values than in cases $\mathrm{B}$ and $\mathrm{C}$. The standard deviations of $\nabla . \vec{u} \times \delta_{t h} / S_{L}$ conditional upon $c$ are greater than the differences in the mean values in cases B and C (not shown here). However, the difference in the mean values of $\nabla . \vec{u} \times \delta_{t h} / S_{L}$ between case A and cases B and C cannot be ignored even after considering the standard deviations conditional on $c$. Figure $6 \mathrm{~b}$ shows significant differences in the mean values of $\nabla \cdot \vec{u} \times \delta_{t h} / S_{L}$ between case $C$ and cases $\mathrm{D}$ and $\mathrm{E}$. The smallest mean value of $\nabla . \vec{u} \times \delta_{t h} / S_{L}$ is obtained for case $\mathrm{C}$ among cases $\mathrm{C}$-E but mean values of $\nabla . \vec{u} \times \delta_{t h} / S_{L}$ for cases D and E remain comparable. The differences in the mean values of $\nabla . \vec{u} \times \delta_{t h} / S_{L}$ between case $\mathrm{C}$ and cases $\mathrm{D}$ and $\mathrm{E}$ remain comparable to the standard deviations between realisations.

The statistical behaviour of tangential strain rate $a_{T}=$ $\nabla . \vec{u}-a_{N}$ is determined by the relative values of dilatation rate $\nabla . \vec{u}$ and normal strain rate $a_{N}$. The variations of mean values of normalised tangential strain rate $a_{T} \times \delta_{t h} / S_{L}$ conditional upon $c$ using the samples for the whole flame are shown in Figs. 7a and 7b for cases A-C and cases C-E respectively. A comparison between Figs. 5 and 6 reveals that the higher mean value of $\nabla \cdot \vec{u} \times \delta_{t h} / S_{L}$ in case A is responsible for higher mean $a_{T} \times \delta_{t h} / S_{L}$ in this case in comparison to cases B and C. Similarly, the higher mean values of $\nabla \cdot \vec{u} \times \delta_{t h} / S_{L}$ in cases $\mathrm{D}$ and $\mathrm{E}$ are responsible for higher mean $a_{T} \times \delta_{t h} / S_{L}$ in these cases in comparison to case C.
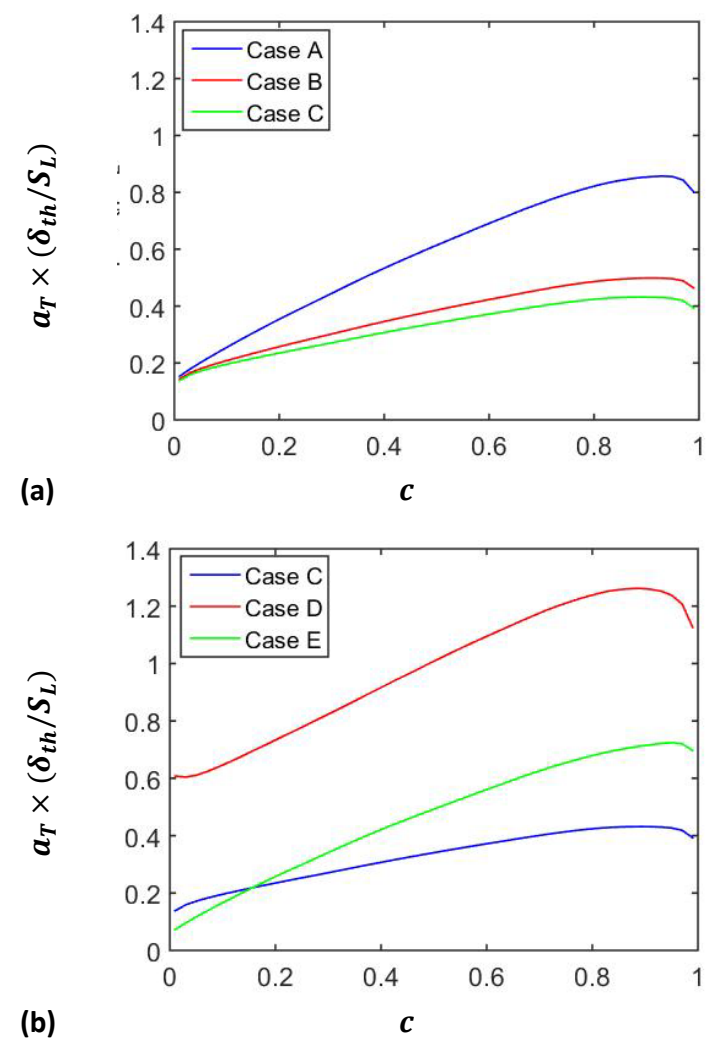

Figure. 7: Profiles of the mean values of normalised fluiddynamic tangential strain rate $a_{T} \times \delta_{t h} / S_{L}$ conditional upon $c$ for cases (a) A-C and (b) C-E.

An explanation is warranted regarding the difference in normalised mean values of dilatation rate. It is well-known [7-9] that dilatation rate is negatively correlated with curvature due to focussing (defocussing) of heat at negatively (positively) curved zones. This is reflected in the negative correlation between $\nabla \cdot \vec{u}$ and curvature $\kappa_{m}$, which can be substantiated from Table 3 where the correlation coefficients between $\nabla \cdot \vec{u}$ and $\kappa_{m}$ are shown for $c=0.8$ isosurface, and the same qualitative trend is obtained for other $c$-isosurfaces. 


\begin{tabular}{|c|c|}
\hline Case & $\boldsymbol{\nabla . \vec { \boldsymbol { u } }}-\boldsymbol{\kappa}_{\boldsymbol{m}}$ \\
\hline A & -0.65 \\
\hline B & -0.60 \\
\hline C & -0.61 \\
\hline D & -0.65 \\
\hline E & -0.57 \\
\hline
\end{tabular}

Table 3: Correlation coefficient between $\nabla \cdot \vec{u}$ and curvature $\kappa_{m}$ on $c=0.8$ isosurface for all cases considered here. Here the mean correlation coefficient based on all the different realisations used here is shown.
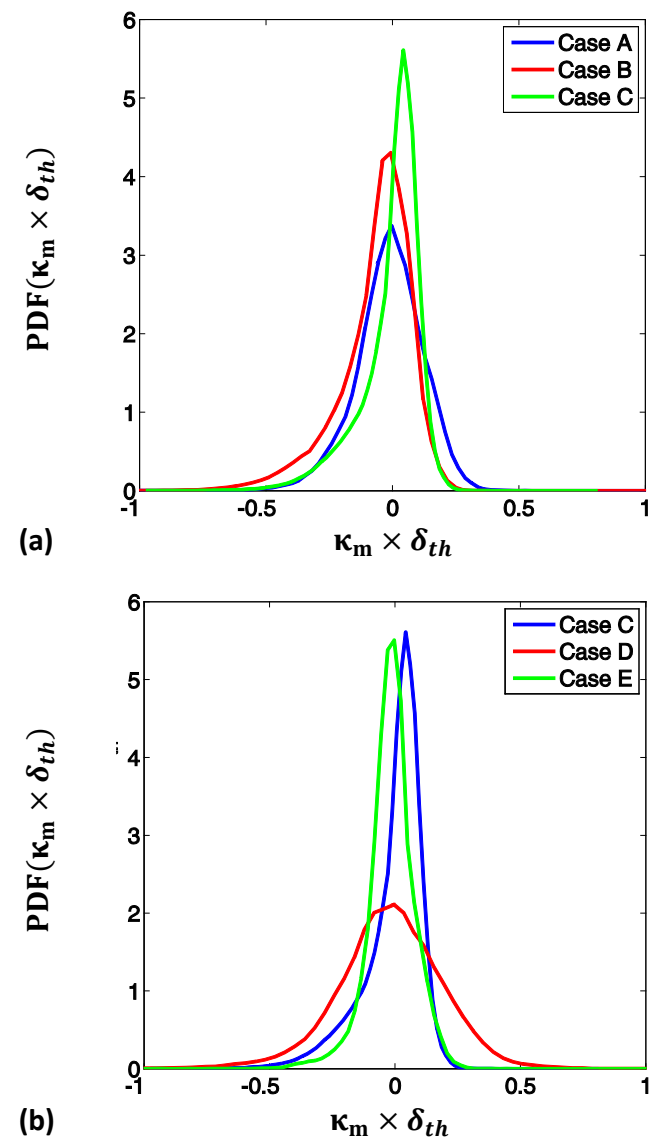

Figure 8: Pdfs of curvature for $c=0.8$ isosurface for cases (a) A-C and (b) C-E.

For the present thermochemistry, the maximum reaction rate takes place close to $c=0.8$. Thus, the curvature pdfs for $c=0.8$ isosurface for cases A-C and cases C-E are shown in Figs. 8a and $8 \mathrm{~b}$ respectively for a given realisation. Figure 8 shows that the flames considered here have negative mean curvatures but the distribution of curvature is significantly different between these cases. A comparison between Figs. 3 and 8 reveals that the cusp like wrinkling becomes increasingly prominent from case A to case C. Figure 8a shows that that the skewness of the curvature pdfs increases from case A to case C. Similarly, Fig. 8b shows greater level of negative skewness in case $\mathrm{C}$ than in cases D and E. Moreover, Fig. 3 demonstrates that the probability of finding cusp like wrinkling decreases from case $\mathrm{C}$ to case $\mathrm{E}$.
Creta et al. [35] suggested that the skewness of the flame curvature pdf is an unambiguous marker for the presence of Darriues-Landau (DL) instability. However, the presence of rounded leading edges toward the unburned mixture and sharp and narrow cusps can occur without the presence of the DL instability due to Huygens principle [36], since the flame surface propagates normal to itself. Shepherd and Ashurst [37] demonstrated that assuming a zero-thickness, constant-density (i.e. absence of heat release and hence the DL instability) premixed flame model compares well with experimental data in terms of flame wrinkling. The effects of the DL instability in thermo-diffusively neutral Bunsen flames are most prominent at an intermediate range of length scales: very small scales are stabilised by the influence of diffusion [38]. Moreover, there is a cutoff wavelength $\lambda_{c}$ such that the flame exhibits the DL instability only for wavelengths larger than $\lambda_{c}$. According to the analytical relation for $\lambda_{c}$ derived in [39], the cutoff wavelength turns out to be proportional to the Markstein length which itself is proportional to the Zeldovich flame thickness $\delta_{Z}$ (i.e. $\delta_{Z}=$ $\alpha_{T 0} / S_{L}$ with $\alpha_{T 0}$ being the thermal diffusivity of the unburned gas). As $\delta_{Z}$ decreases with increasing pressure the DL instability becomes more likely with increasing pressure. As shown in Table 4, the dispersion relations in $[39,40]$ suggest that $\lambda_{c}$ is clearly smaller than the nozzle diameter in the elevated pressure cases B and C, whereas it is close to $D$ for cases A, D and E. For the DL instability to occur, a disturbance of wavelength $\lambda>\lambda_{c}$ is required. Accurate determination of instability criterion for the DL instability is beyond the scope of this paper but it is clear that the effects of the DL instability are highly likely in the high-pressure case $\mathrm{C}$, and to some extent in case B. However, Huygens propagation alone is responsible for the negative skewness in curvature pdfs in cases A, D and E [36]. The effects of Huygens propagation and negative skewness in the curvature pdf are least prominent in case $E$ because of its larger integral length scale in comparison to the other cases. A more detailed discussion regarding the characterization of flame curvature in high pressures Bunsen flames can be found in [36].

\begin{tabular}{|c|c|c|c|}
\hline $\boldsymbol{\lambda}_{\boldsymbol{c}} / \boldsymbol{D}$ & $\boldsymbol{P} / \boldsymbol{P}_{\mathbf{0}}=\mathbf{1}$ & $\boldsymbol{P} / \boldsymbol{P}_{\mathbf{0}}=\mathbf{5}$ & $\boldsymbol{P} / \boldsymbol{P}_{\mathbf{0}}=\mathbf{1 0}$ \\
\hline$[40]$ & 0.96 & 0.42 & 0.30 \\
\hline$[39]$ & 0.50 & 0.23 & 0.15 \\
\hline
\end{tabular}

Table 4: Critical wave length normalized by the nozzle diameter for three different pressure levels according to Creta \& Matalon [40] and Matalon \& Matkowsky [39].

The presence of the DL instability in case C acts to increase the probability of finding local positive values of $\kappa_{m}$ along with a long tail on the negative side for very high negative curvature $\kappa_{m}$ values. The negative correlation between $\nabla . \vec{u}$ and curvature $\kappa_{m}$ is non-linear in nature which can be substantiated from the non-unity magnitude of the correlation coefficient between $\nabla . \vec{u}$ and $\kappa_{m}$ listed in Table 3 . The high probability of finding positive curvature $\kappa_{m}$ acts to reduce the mean dilatation rate in case $\mathrm{C}$ due to defocusing of heat ahead of the positively curved bulges. Although high magnitudes of dilatation rate can be obtained at sharply negatively curved cusps in case C, Fig. 8 shows the frequency of obtaining sharply negatively curved cusps is smaller than the one for positively curved bulges. As a result the effects of reduced dilatation rate at positively curved bulges eclipse the high dilatation rate values at negatively curved cusps in case $\mathrm{C}$ due to non- 
linear correlation between $\nabla . \vec{u}$ and curvature $\kappa_{m}$. This gives rise to a smaller mean value of dilatation rate $\nabla . \vec{u}$ in case $\mathrm{C}$ than in the other cases. Thus, the differences in the mean behaviours of $\nabla . \vec{u}$ and $a_{T}$ in Figs. 6 and 7 are not direct effects of pressure and turbulent Reynolds number variations but arise due to the DL instability, which becomes more likely for higher values of pressure due to the reduction in flame thickness.
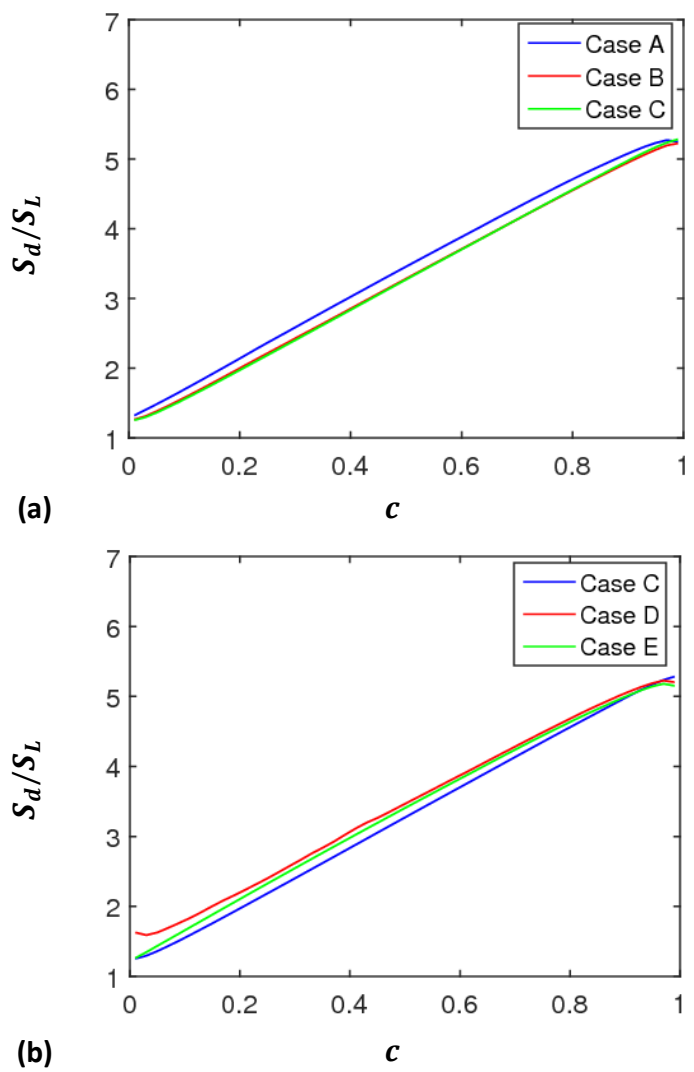

Figure. 9: Profiles of the mean values of normalised displacement speed $S_{d} / S_{L}$ conditional upon $c$ for cases (a) A-C and (b) C-E.

4.4 Statistical behaviour of flame displacement speed The variations of mean values of normalised flame displacement speed $S_{d} / S_{L}$ conditional upon $c$ using the samples for the whole flame are shown in Figs. 9a and 9b for cases A-C and C-E respectively. It is clear from Fig. 9 that mean $S_{d} / S_{L}$ assumes positive values and increases from unburned to burned gas side of the flame due to thermal expansion (or density drop). Moreover, the mean values of $S_{d} / S_{L}$ remain close to each other for cases A-E and the standard deviation of $S_{d} / S_{L}$ conditional upon $c$ remains much greater than the difference in mean values of normalised displacement speed. Thus, the small differences in the mean values of $S_{d} / S_{L}$ cannot be attributed to any physical mechanism but physical explanations are warranted for the similar behaviour of mean $S_{d} / S_{L}$ for the cases considered here. In order to explain the observed mean behaviour of $S_{d} / S_{L}$ the variations of mean values of normalised displacement speed components $S_{r} / S_{L}, S_{n} / S_{L}$ and $S_{t} / S_{L}$ conditional upon $c$ using the samples for the whole flame are shown in Fig. 10 for case B. Similar qualitative behaviours have been observed in all other cases, and thus are not shown here for the sake of brevity.

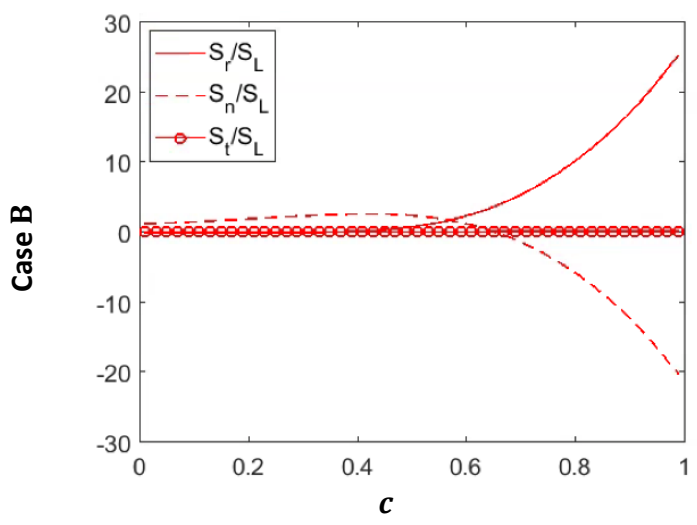

Figure 10: Profiles of the mean values of normalised displacement speed components $S_{r} / S_{L}, S_{n} / S_{L}$ and $S_{t} / S_{L}$ conditional upon $c$ for case B.

\begin{tabular}{|c|c|c|}
\hline Case & $\boldsymbol{S}_{\boldsymbol{d}}-\boldsymbol{\kappa}_{\boldsymbol{m}}$ & $|\boldsymbol{\nabla c}|-\boldsymbol{\kappa}_{\boldsymbol{m}}$ \\
\hline A & -0.72 & 0.22 \\
\hline B & -0.92 & 0.10 \\
\hline C & -0.91 & 0.14 \\
\hline D & -0.60 & 0.07 \\
\hline E & -0.79 & 0.24 \\
\hline
\end{tabular}

Table 5: Correlation coefficients between curvature $\kappa_{m}$ and displacement speed $S_{d}$ and between curvature $\kappa_{m}$ and SDF $|\nabla c|$. Here the mean correlation coefficient based on all the different realisations is shown.

\begin{tabular}{|c|c|c|}
\hline Case & $\boldsymbol{a}_{\boldsymbol{T}}-\boldsymbol{\kappa}_{\boldsymbol{m}}$ & $\boldsymbol{a}_{\boldsymbol{N}}-\boldsymbol{\kappa}_{\boldsymbol{m}}$ \\
\hline $\mathbf{A}$ & -0.77 & -0.12 \\
\hline $\mathbf{B}$ & -0.91 & -0.03 \\
\hline $\mathbf{C}$ & -0.93 & -0.00 \\
\hline $\mathbf{D}$ & -0.79 & -0.16 \\
\hline $\mathbf{E}$ & -0.67 & -0.17 \\
\hline
\end{tabular}

Table 6: Correlation coefficients between curvature $\kappa_{m}$ and different strain rates (i.e. $a_{T}$ and $a_{N}$ ). Here the mean correlation coefficient based on all the different realisations is shown.

The normalised reaction component of displacement speed $S_{r} / S_{L}$ assumes positive values due to positive values of $\dot{w} / \rho$ and $|\nabla c|$. The mean value of normal diffusion component of displacement speed $S_{n}$ remains positive with small magnitude towards the unburned gas side but it becomes negative towards the burned gas side. This behaviour originates due to mean variation of $\vec{N} \cdot \nabla\left(\rho D_{c} \vec{N} \cdot \nabla c\right)=-\partial\left(\rho D_{c}|\nabla c|\right) / \partial x_{N}$, which shows positive (negative) mean value towards the unburned (burned) gas side of the flame $[7,8,41,42]$. It can be seen from Fig. 11 that the mean value of $S_{t} / S_{L}$ remains negligible in comparison to its mean values of $S_{r} / S_{L}$ and $S_{n} / S_{L}$. Although $S_{t}$ does not significantly contribute to the mean $S_{d}$, the influence of $S_{t}$ remains significant to the local variation of displacement speed. The results in Fig. 10 indicate that both pressure and turbulent Reynolds number variations do not significantly affect the mean behaviour of displacement speed and its components. 


\subsection{Modelling implications}

The mean behaviours of strain rates and SDF have been discussed in the previous sub-sections but also the local curvature and strain rate dependences of $|\nabla c|$ and $S_{d}$ remain qualitatively similar for all cases considered here. This can be substantiated from the correlation coefficients listed in Table 5. Similarly, local curvature dependences of $a_{T}$ and $a_{N}$, remain qualitatively similar for all cases considered here which can be substantiated from Table 6 . The joint pdfs corresponding to the correlation coefficients listed in Tables 5 and 6 are not shown here for the sake of conciseness. Tables 5 and 6 indicate that the variations of pressure and turbulent Reynolds number do also not affect the qualitative nature of local behaviours of $|\nabla c|, S_{d}, a_{T}$ and $a_{N}$ for the ranges considered here.

The transport equation of $|\nabla c|$ can be written by expanding eq. 2 :

$\frac{\partial|\nabla c|}{\partial t}+\frac{\partial\left(u_{j}|\nabla c|\right)}{\partial x_{j}}=a_{T}|\nabla c|+2 S_{d} \kappa_{m}|\nabla c|-\frac{\partial\left(S_{d} N_{j}|\nabla c|\right)}{\partial x_{j}}$

It is possible to obtain the transport equation of the generalised FSD (i.e. $\Sigma_{g e n}=\overline{|\nabla c|}$ ) on Reynolds averaging/LES filtering eq.5 [3]. Multiplying eq. 5 by $2|\nabla c|$ yields:

$$
\begin{gathered}
\frac{\partial|\nabla c|^{2}}{\partial t}+u_{j} \frac{\partial|\nabla c|^{2}}{\partial x_{j}}= \\
-2 a_{N}|\nabla c|^{2}-2 N_{j} \frac{\partial S_{d}}{\partial x_{j}}|\nabla c|^{2}-S_{d} N_{j} \frac{\partial|\nabla c|^{2}}{\partial x_{j}}
\end{gathered}
$$

Algebraic manipulation of eq. 6 provides the transport equation of scalar dissipation rate (SDR) $N_{c}=D_{c}|\nabla c|^{2}$ :

$$
\begin{aligned}
& \frac{\partial\left(\rho N_{c}\right)}{\partial t}+\frac{\partial\left(\rho u_{j} N_{c}\right)}{\partial x_{j}}=-2 \rho a_{N} N_{c}-2 \rho N_{j} \frac{\partial S_{d}}{\partial x_{j}} N_{c}-\rho S_{d} N_{j} \frac{\partial N_{c}}{\partial x_{j}}+ \\
& \rho S_{d} N_{j} N_{c} \frac{1}{D_{c}} \frac{\partial D_{c}}{\partial x_{j}}+\frac{\rho N_{c}}{D_{c}}\left(\frac{\partial D_{c}}{\partial t}+u_{j} \frac{\partial D_{c}}{\partial x_{j}}\right)
\end{aligned}
$$

It is evident from eqs. 5-7 that $a_{N}, a_{T}$, and $S_{d}$ play key roles in both the FSD and SDR transport. The present analysis suggests that the variation of pressure does not have significant influences on the statistical behaviours of $|\nabla c| \times \delta_{t h}, a_{N} \times \delta_{t h} / S_{L}$, and $S_{d} / S_{L}$. However, the DL instability at high pressure flames affects the statistics of $a_{T} \times \delta_{t h} / S_{L}$. Thus, existing algebraic closures of FSD and SDR, and the sub-models for FSD and SDR transport, which are related to $a_{N}$, may also work for elevated pressure conditions at least in the flamelet regime of combustion. However, the models for the terms related to $a_{T}$ and $\kappa_{m}$ need to account for the effects of the DL instability for flames at elevated pressures.

\section{CONCLUSIONS}

Three-dimensional compressible simple chemistry DNS simulations of turbulent Bunsen burner flames have been carried out for different pressure levels. Three cases (i.e. cases A-C) with different pressures have been considered for a given set of values of normalised mean inlet velocity $U_{\text {mean }} / S_{L}$, normalised root-mean square turbulent velocity fluctuation at the inlet $u^{\prime} / S_{L}$ and longitudinal integral length scale of inlet turbulence normalised by nozzle diameter $L_{11} / D$. However, changes in $S_{L}$ and $\delta_{t h}$ with pressure give rise to modifications in Damköhler number $\mathrm{Da}$, Karlovitz number $\mathrm{Ka}$ and turbulent Reynolds number $R e_{t}$. Two more cases (i.e. case $\mathrm{D}$ and $\mathrm{E}$ ) at the lowest pressure value have been considered such that they match the turbulent Reynolds number of the inlet stream for the highest pressure case. The turbulent Reynolds numbers for these cases have been matched to that in the highest pressure case (i.e. case $\mathrm{C}$ ) by modifying only $u^{\prime} / S_{L}$ in case $\mathrm{D}$ and by changing only $L_{11} / D$ in case E. All the cases considered here represent combustion in the flamelet regime. It has been found that the flamelet thickness of turbulent flames remains almost the same as that of the laminar flame thickness for all cases considered here. Both pressure and turbulent Reynolds number variations have no significant influences on the mean behaviours of fluid-dynamic normal strain rate, displacement speed and its components. The DarrieusLandau (DL) instability becomes more likely to occur for high pressure flames and thus the flames at high pressure exhibits negatively curved cusps and positively curved bulges which are characteristics of the DL instability. The presence of the DL instability is manifested in the form of high negative skewness in curvature pdfs. Although, Huygens propagation can also lead to a negative skewness in the curvature pdfs in the absence of the DL instability, the presence of this instability increases the negative skewness of curvature pdfs. The probability of positively curved bulges acts to reduce local dilatation rate due to defocussing of heat and thus the high pressure flames with the DL instability exhibit smaller mean values of dilatation rate and tangential strain rate than the low pressure flames without the DL instability. It has been found that the flame surface area normalised by $D^{2}$ assumes similar values in cases A-D but slightly smaller values are obtained for case $\mathrm{E}$ due to the absence of cusp formation and low turbulence intensity which is accompanied by small amount of flame wrinkling. The similarity in the statistical behaviours of $|\nabla c|, a_{N}$ and $S_{d}$ implies that existing models for the unclosed terms related to these quantities in the context of Flame Surface Density (FSD) and Scalar Dissipation Rate (SDR) closures, which have been validated with respect to atmospheric pressure conditions are likely to be valid even for elevated pressures in the absence of the DL instability. However, the DL instability is likely to occur at high pressure conditions as the length scale separation given by $D / \delta_{t h}$ increases with increasing pressure for a given Bunsen burner, and this is expected to influence the tangential strain rate and dilatation rate contributions to the FSD and SDR transports. Thus, the models for the tangential strain rate and dilatation rate contributions to the FSD and SDR transports need to account for the effects of the DL instability in order to obtain high-fidelity closures for elevated pressure conditions. The statistics of the SDF $|\nabla c|$ transport for the database considered here has been analysed in a complementary work [43].

It is worth noting that the present analysis simplifies the chemical mechanism for the purpose of computational economy. Thus, the current analysis focuses only on fluiddynamical aspects of the influences of pressure on scalar gradient and displacement speed statistics. Although several previous analyses demonstrated at least qualitative similarities between the displacement speed [21,22,41, 42, $44,45]$ and scalar gradient $[4,8,9,46]$ statistics between simple and detailed chemistry DNS results, future analyses with detailed chemistry and transport will be necessary for validation of current findings. Moreover, the pressure variation may give rise to differences in pollutant emission, which cannot be addressed without detailed chemistry based DNS simulations. Some of these 
aforementioned aspects will be addressed in future investigations.

\section{ACKNOWLEDGEMENTS}

The authors are grateful to Gauss Centre for Supercomputing / Leibniz Supercomputing Centre (grant: pr74ra) and ARCHER (EP/K025163/1) for computing support.

\section{REFERENCES}

[1] K. N. C. Bray and N. Swaminathan, Scalar dissipation rate and flame surface density in premixed turbulent combustion, C. R. Mech., 334 (2006) 466-473.

[2] W. Kollmann, J.H. Chen, Pocket formation and the flame surface density equation, Proc. Combust. Inst., 27 (1998) 927-934.

[3] M. Boger, D. Veynante, H. Boughanem, A. Trouvé, Direct Numerical Simulation analysis of flame surface density concept for Large Eddy Simulation of turbulent premixed combustion, Proc. Combust. Inst., 27 (1998) 917-925.

[4] N. Chakraborty, R.S. Cant, Effects of strain rate and curvature on Surface Density Function transport in turbulent premixed flames in the thin reaction zones regime, Phys. Fluids, 17 (2005) 65108.

[5] S. H. Kim, H., Pitsch, Scalar gradient and small-scale structure in turbulent premixed combustion, Phys. Fluids, 19 (2007) 115104.

[6] R. Sankaran, E.R. Hawkes, J.H. Chen, T. Lu, C.K. Law, Structure of a spatially developing turbulent lean methane-air Bunsen flame, Proc. Combust. Inst., 31 (2007) 1291-1298.

[7] N. Chakraborty, M. Klein, Influence of Lewis number on the Surface Density Function transport in the thin reaction zones regime for turbulent premixed flames, Phys. Fluids, 20 (2008) 065102.

[8] N. Chakraborty, E.R. Hawkes, J.H. Chen, R.S. Cant, Effects of strain rate and curvature on Surface Density Function transport in turbulent premixed $\mathrm{CH}_{4}$-air and $\mathrm{H}_{2}-$ air flames: A comparative study, Combust. Flame, 154 (2008) 259-280.

[9] N. Chakraborty, M. Klein, Effects of global flame curvature on the Surface Density Function transport in Turbulent premixed flame kernels in the Thin Reaction Zones regime.” Proc. Combust. Inst., 32 (2009) 14351443.

[10] N. Chakraborty, N. Swaminathan, Influence of Damköhler number on turbulence-scalar interaction in premixed flames, Part I: Physical Insight, Phys. Fluids, 19 (2007) 045103.

[11] N. Chakraborty, M. Klein, N. Swaminathan, Effects of Lewis number on reactive scalar gradient alignment with local strain rate in turbulent premixed flames, Proc. Combust. Inst., 32 (2009) 1409-1417.

[12] G. Hartung, J. Hult, C.F. Kaminski, J.W. Rogerson, $\mathrm{N}$. Swaminathan, Effect of heat release on turbulence and scalar-turbulence interaction in premixed combustion, Phys. Fluids, 20 (2008) 035110.

[13] L. Cifuentes, C. Dopazo, J. Martin, C. Jimenez, Local flow topologies and scalar structures in a turbulent premixed flame, Phys. Fluids, 26 (2014) 065108.

[14] L. Cifuentes, C. Dopazo, J. Martin, P. Domingo, L. Vervisch, Local volumetric dilatation rate and scalar geometries in a premixed methane-air turbulent jet flame, Proc. Combust. Inst., 35 (2015) 1295-1303.

[15] C. Dopazo, L. Cifuentes, J. Martin, C. Jimenez, Strain rates normal to approaching isoscalar surfaces in a turbulent premixed flame, Combust. Flame, 162 (2015) 1729- 1736.

[16] C. Dopazo, L. Cifuentes, J. Hierro, J. Martin, J., Micro-scale mixing in turbulent constant density reacting flows and premixed combustion, Flow Turb. Combust., 96 (2015) 547-571.

[17] C. Dopazo, L. Cifuentes, The physics of scalar gradients in turbulent premixed combustion and its relevance to modeling, Combust. Sci. Technol., 188(9) (2016) 1376-1397.

[18] H. Wang, E.R. Hawkes, J. H. Chen, B. Zhou, Z. Li, M. Alden, Direct numerical simulations of a high Karlovitz number laboratory premixed jet flame- an analysis of flame stretch and flame thickening, J. Fluid Mech., 815 (2017) 511-536.

[19] S.R. Turns, An introduction to combustion: concepts and applications, $3^{\text {rd }}$ Edition, McGraw Hill (2011).

[20] J.H. Chen, A. Choudhary, M. de Supinski, B. de Vries, E.R. Hawkes, S. Klasky, W.K. Liao, K.L. Ma, J. Mellor-Crummey, N. Podhorski, R. Sankaran, S. Shende and C. Yoo, Terascale direct numerical simulations of turbulent combustion using S3D. Comput. Sci. Discov., 2 (2009) 015001.

[21] N. Peters, P. Terhoeven, J.H. Chen, T. Echekki, Statistics of Flame Displacement Speeds from Computations of 2-D Unsteady Methane-Air Flames, Proc. Combust. Inst., 27 (1998) 833-839.

[22] T. Echekki, J.H. Chen, Analysis of the contribution of curvature to premixed flame propagation, Combust. Flame, 118 (1999) 308-311.

[23] K.W. Jenkins, R.S. Cant, DNS of turbulent flame kernels, In C. Liu, L. Sakell and T. Beautner (Eds.), Proc. $2^{\text {nd }}$ AFOSR Conf. on DNS and LES, Kluwer Academic Publishers, (1999) 192-202.

[24] N. Peters, Turbulent Combustion, Cambridge Monograph on Mechanics, Cambridge University Press, Cambridge (2000).

[25] M. Klein, A. Sadiki , J. Janicka, A digital filter based generation of inflow data for spatially developing direct numerical or large eddy simulations, J. Comp. Phys., 186 (2003) 652-665.

[26] N. Jarrin, S. Benhamadouche, D. Laurence, R. Prosser, A synthetic-eddy-method for generating inflow conditions for large-eddy simulations, Int. J. Heat and Fluid Flow, 27 (2006) 585-593.

[27] Z.-T. Xie, I.P. Castro, Efficient Generation of Inflow Conditions for Large Eddy Simulation of Street-Scale Flows, Flow, Turb. Combust., 81 (2008) 449-470.

[28] A.M. Kempf, S. Wysocki, M. Pettit, An efficient, parallel low-storage implementation of Klein's turbulence generator for LES and DNS, Comput. Fluids 60 (2012) $58-60$.

[29] T. Poinsot, S.K. Lele, Boundary conditions for direct simulation of compressible viscous flows, J. Comp. Phys. 101 (1992) 104-129.

[30] A. Soika, F. Dinkelacker, A. Leipertz, Measurement of resolved flame structure with constant Reynolds number, Proc. Combust. Inst., 27 (1998) 785-792.

[31] E.R. Hawkes, J. H. Chen, Direct numerical simulation of hydrogen-enriched lean premixed methaneair flames, Combust. Flame, 138 (2004) 242-258.

[32] F. O'Young, R.W. Bilger, Scalar gradient and related quantities in turbulent premixed flames, Combust. Flame, 109 (1997) 683-700.

[33] Y.-C. Chen, M. S. Monsour, Investigation of flame broadening in the thin reaction zones regime, Proc. Combust. Inst., 27 (1998) 811-818. 
[34] Y.-C. Chen, R. W. Bilger, Experimental investigation of three-dimensional fame front structure in premixed turbulent combustion-I: hydrocarbon/air bunsen fames, Combust. Flame, 131 (2002) 400-435.

[35] F. Creta, R. Lamioni, P.E. Lapenna, G. Troiani, Interplay of Darrieus-Landau instability and weak turbulence in premixed flame propagation Physical Review E 94 (2016) 053102.

[36] M. Klein, H. Nachtigal, M. Hansinger, M. Pfitzner and N. Chakraborty, Flame Curvature in High Pressure Bunsen Flames, Proceedings of the $10^{\text {th }}$ Mediterranean Combust. Symp. Naples (2017).

[37] I.G. Shepherd, W.T. Ashurst, Flame front geometry in premixed turbulent flames, Proc. Combust. Inst., 24 (1992) 485-491.

[38] F. Creta, M. Matalon, Propagation of wrinkled turbulent flames in the context of hydrodynamic theory, $\mathrm{J}$. Fluid Mech., 680 (2011) 225-264.

[39] M. Matalon, B.J. Matkowsky, Flames as gasdynamic discontinuities, J. Fluid Mech., 124 (1982) 239-259.

[40] F. Creta, M. Matalon, Strain rate effects on the nonlinear development of hydrodynamically unstable flames, Proc. Combust. Inst., 33 (2011) 1087-1094.

[41] N. Chakraborty and S. Cant, Unsteady effects of strain rate and curvature on turbulent premixed flames in an inlet-outlet configuration, Combust. Flame, 137 (2004) 129-147.

[42] N. Chakraborty, R.S. Cant, Influence of Lewis Number on curvature effects in turbulent premixed flame propagation in the thin reaction zones regime, Phys. Fluids, 17 (2005) 105105.

[43] M. Klein, D. Alwazzan, N. Chakraborty, A Direct Numerical Simulation analysis of pressure variation in turbulent premixed Bunsen burner flames-Part 2: Surface Density Function transport statistics Comput. Fluids (under review).

[44] N. Chakraborty, M. Klein, R.S. Cant, Stretch rate effects on displacement speed in turbulent premixed flame kernels in the thin reaction zones regime, Proc. Combust. Inst., 31 (2007) 1385-1392.

[45] N. Chakraborty, M. Klein, R.S. Cant, Effects of turbulent Reynolds number on the displacement speed statistics in the thin reaction zones regime turbulent premixed combustion, J. Combust., 2011 (2011) 473679. [46] N. Chakraborty, H. Kolla, R. Sankaran, E. R. Hawkes, J. H. Chen, N. Swaminathan, Determination of three-dimensional quantities related to scalar dissipation rate and its transport from two-dimensional measurements: Direct Numerical Simulation based validation, Proc. Combust. Inst., 34 (2013)1151-1162. 
Original Word File
Click here to download LaTeX Source Files: Bunsen_strain_part1_final_NC_MK_3rdFeb.docx

Click here to download LaTeX Source Files: Bunsen_strain_part1_final_NC_MK_3rdFeb.docx

(a)

(1)

(1)

$\sqrt{20}$

-

-

列

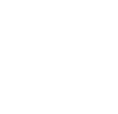

